\title{
Biosynthesis of Chondroitin in Engineered Corynebacterium glutamicum ${ }^{\text {s }}$
}

\author{
Fangyu Cheng ${ }^{1,2}$, Sijin Luozhong ${ }^{1,2}$, Huimin $\mathrm{Yu}^{1,2,3 *}$, and Zhigang Guo ${ }^{1}$ \\ ${ }^{1}$ Department of Chemical Engineering, Tsinghua University, Beijing 100084, P.R. China \\ ${ }^{2}$ Key Laboratory of Industrial Biocatalysis (Tsinghua University), the Ministry of Education, Beijing 100084, P.R. China \\ ${ }^{3}$ Center for Synthetic and Systems Biology, Tsinghua University, Beijing 100084, P.R. China
}

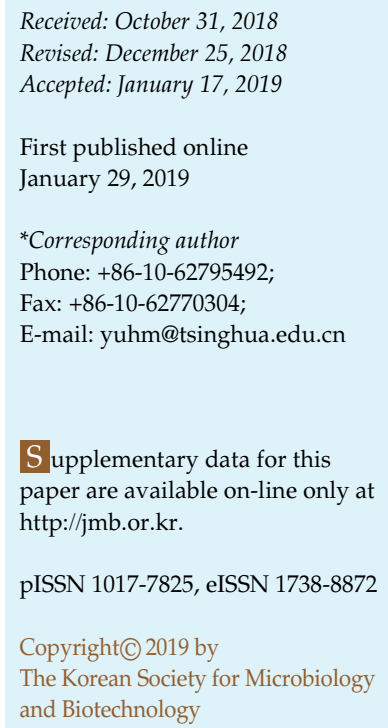

\begin{abstract}
Chondroitin, the precursor of chondroitin sulfate, which is an important polysaccharide, has drawn significant attention due to its applications in many fields. In the present study, a heterologous biosynthesis pathway of chondroitin was designed in a GRAS (generally recognized as safe) strain $C$. glutamicum. $C g k f o C$ and $C g k f o A$ genes with host codon preference were synthesized and driven by promoter Ptac, which was confirmed as a strong promoter via GFPuv reporter assessment. In a lactate dehydrogenase (ldh) deficient host, intracellular chondroitin titer increased from 0.25 to $0.88 \mathrm{~g} / 1$ compared with that in a wild-type host. Moreover, precursor enhancement via overexpressing precursor synthesizing gene ugdA further improved chondroitin titers to $1.09 \mathrm{~g} / 1$. Chondroitin production reached $1.91 \mathrm{~g} / 1 \mathrm{l}$ with the engineered strain C. glutamicum $\triangle \mathrm{L}-\mathrm{CgCAU}$ in a 5-L fed-batch fermentation with a single distribution $\mathrm{M}_{\mathrm{w}}$ of $186 \mathrm{kDa}$. This work provides an alternative, safe and novel means of producing chondroitin for industrial applications.
\end{abstract}

Keywords: Chondroitin biosynthesis, engineered Corynebacterium glutamicum, lactate dehydrogenase deficient, precursor enhancement, fed-batch fermentation

\section{Introduction}

Chondroitin sulfate (CS) is an essential glycosaminoglycan (GAG) located in mammalian extracellular matrices [1]. Due to its diverse biological and physiological functions, CS has been widely investigated and applied in clinical fields, such as an anti-inflammatory drug for treating osteoarthritis and rheumatism, in cartilage disease treatment, in cancer diagnoses and treatment, and tissue scaffold building with other biomacromolecules (e.g., proteoglycan and hyaluronic acid) [2-4]. As the world population increasingly ages, the market demand for CS has correspondingly increased.

CS is conventionally extracted from animal tissues, such as shark fins and bovine trachea. While seemingly industrially feasible, the reliance on an animal-derived production process has a number of drawbacks, including low productivity, costly downstream processing and harmful environmental impacts [5]. Additionally, there are growing concerns for potential risks of interspecies viral transmission. In response, researchers have turned to microbial production as a safer and more reliable alternative. Chondroitin, containing $\beta$-D-glucuronic acid (GlcUA) and $\mathrm{N}$-acetyl-D-galactosamine (GalNAc) as a disaccharide unit, is the backbone of CS and shares structural similarity with other GAGs (Fig. S1). Chemo-enzymatic sulfation of microbially produced chondroitin has become an attractive approach to producing CS [6-9].

A capsular polysaccharide (CPS) of Escherichia coli K4 has been discovered as an unsulfated chondroitin with a fructose residue at the 3-position of GlcUA [10]. The similarity of K4CPS (fructose chondroitin) to chondroitin allows for potential CS production by microbial fermentation. Through defructosylation and sulfation, K4CPS could be further converted into CS [11]. Researchers have explored the biochemical basis of fructose chondroitin synthesis in bacteria and have identified enzymes involved in this process [6]. Cimini et al. enhanced K4CPS production 
by genetic engineering and bioprocess optimization. They achieved K4CPS titers of $3.5 \mathrm{~g} / \mathrm{l}$ by replacing transposase with chondroitin polymerase [12]. Recently, $\mathrm{Wu}$ et al. attempted to balance the metabolic flux of intracellular K4CPS precursors UDP-GalNAc and UDP-GlcUA and obtained an $8.4 \mathrm{~g} / \mathrm{l}$ titer (the highest level achieved to date) in a 30-L fermentor by glycerol and dissolved oxygen-stat feeding [13]. Despite these promising results, E. coli K4 is a pathogenic bacteria and may cause urinary tract infections [14]. Thus, an alternative chondroitin production pathway in a safer host was considered.

He et al. constructed a plasmid system with a pseudooperon containing gene $k f o C-k f o A-k f o F$ in E. coli BL21, and achieved a $2.4 \mathrm{~g} / 1$ intracellular chondroitin titer in a dissolved oxygen-stat fed-batch bioreactor [15]. Bacillus subtilis, regarded as a GRAS (generally recognized as safe) strain, is also an alternative in the heterogeneous production of chondroitin. Jin et al. utilized B. subtilis 168 as a host and successfully produced extracellular chondroitin by integrating expression cassette PxylA-kfoC-kfoA into the genome and enhanced the biosynthesis of chondroitin by tuaD upregulation. Titers of $2.54 \mathrm{~g} / 1$ and $5.22 \mathrm{~g} / 1$ extracellular chondroitin were accumulated in shaking flask culture and 3-L fermentor, respectively. The weightaverage molecular weights $\left(\mathrm{M}_{\mathrm{w}}\right)$ of these products were $114.07 \mathrm{kDa}$ in the shaking flask and $65.93 \mathrm{kDa}$ in the 3-L fermentor. Although the results showed that B. subtilis 168 was able to transport chondroitin to the extracellular matrix, the mechanism by which this occurred remained unclear [16]. Zhou et al. further compared operon structures among different enzymes responsible for the biosynthesis of chondroitin precursors and obtained $7.15 \mathrm{~g} / 1$ chondroitin in engineered B. subtilis 168 after $70 \mathrm{~h}$ cultivation in a 3-L fermentor [17].

Corynebacterium glutamicum is a Gram-positive strain free of exotoxins and endotoxins. As a GRAS strain, it is an excellent host to produce food and drug related products, such as organic acids and amino acids [18-21]. In our previous study, C. glutamicum was engineered to efficiently biosynthesize hyaluronic acid (HA), a GAG similar to chondroitin [22, 23]. Moreover, C. glutamicum is a nonsporulating strain that will not suffer from spore formation at the late stage of fermentation as B. subtilis.

In the present research, we attempted to biosynthesize chondroitin in the novel host C. glutamicum via several strategies, i.e., pathway engineering, promoter optimization, byproduct gene deletion and precursor upregulation. Finally, a fed-batch culture of recombinant C. glutamicum was conducted in a 5 -L fermentor to produce chondroitin.

\section{Material and Methods}

\section{DNA Manipulation}

Plasmid DNA, agarose gel electrophoresis, restriction enzyme digestion, DNA ligation and DNA transformation were performed following standard protocols [24] or manufacturer instructions. Phanta DNA polymerase used in PCR was purchased from Vazyme Biotech Co., Ltd. (China). Gel extraction kit and plasmid miniprep kits were purchased from Omega. QuickCut restriction enzymes were purchased from Takara.

\section{Gene, Plasmid Vector and Bacterial Strain}

Gene cluster $\mathrm{CgkfoC}-\mathrm{CgkfoA}$ containing RBS sequence was synthesized by Qinglan Biotech Co., Ltd. (Wuxi, China), with $\mathrm{XbaI} / \mathrm{KpnI}$ restriction sites using primers C-F and A-R. Gene $u g d A$ was cloned from the genome of C. glutamicum ATCC13032 using primers U-F and U-R with the restriction sites KpnI/SacI. Table S1 in Supporting Information lists all primers used in this study.

Plasmid pXMJ19 harboring inducible promoter Ptac served as the backbone of the vector. All recombinant plasmids were constructed by inserting the target genes into the vector and are summarized in Supporting Information, Table S2.

C. glutamicum- $\Delta l d h$ was constructed via double crossover homologous recombination driven by negative selection marker gene sacB [25]. Both C. glutamicum ATCC13032 and C. glutamicum$\Delta l d h$ were used for expressing the chondroitin biosynthesis operon. E. coli Top 10 (Solarbio) was used for cloning a shuttle vector and its driven plasmids. All wild-type and engineering strains are summarized in Supporting Information, Table S2.

\section{Codon Usage Preference Analysis}

The codon usage preference of $C$. glutamicum was based on the Kazusa online database (http://www.kazusa.or.jp/codon/cgibin $/$ showcodon .cgi? species $=196627 \& a a=1 \&$ style $=N$ ). Statistical analyses of the rare codons were conducted via a graphical codon usage analyzer (http:/ /gcua.schoedl.de/sequential_v2.html).

\section{Chondroitin Titer and Weight-Average Molecular Weight Measurement}

Fermentation broth was centrifuged at $4^{\circ} \mathrm{C}$ and $10,000 \times g$ to separate cell pellets and supernatant. To determine intracellular chondroitin concentration, cell pellets were collected and redissolved in deionized water. Then, the intracellular contents were released via an Ultra-High Pressure Continuous Flow Cell Disrupter JN-02C (JNBIO, Guangzhou, China). The supernatant was collected and centrifuged at $4^{\circ} \mathrm{C}, 10,000 \times g$ to remove cell fragments. To purify the chondroitin, intracellular supernatant was precipitated using 3 volumes of ethanol and incubated at $4{ }^{\circ} \mathrm{C}$ for $3 \mathrm{~h}$. The recovered sediments were redissolved in deionized water for further chondroitin titer measurement. To determine extracellular chondroitin concentrations, supernatants from the fermentation broth were mixed with 3 volumes of ethanol to precipitate chondroitin at $4^{\circ} \mathrm{C}$ for $3 \mathrm{~h}$. Precipitated chondroitin 
was redissolved in deionized water for further chondroitin titer measurement.

A modified uronic acid carbazole assay [26] was applied to determine intracellular and extracellular chondroitin titers. Briefly, a $0.5 \mathrm{ml}$ sample was added to $3 \mathrm{ml}$ sulfuric acid reagent ( $9.5 \mathrm{~g} / 1$ sodium tetraborate dissolved in sulfuric acid) and heated in boiling water for $20 \mathrm{~min}$. Then, $0.1 \mathrm{ml}$ carbazole reagent ( $1.25 \mathrm{~g} / 1$ carbazole dissolved in ethanol) was added and heated for another $15 \mathrm{~min}$. Finally, $\mathrm{OD}_{530}$ was measured to determine the chondroitin titer according to the standard curve. C. glutamicum with empty plasmid was used as a blank control.

Gel permeation chromatography (GPC) combined with a differential refraction detector was applied to measure chondroitin $\mathrm{M}_{\mathrm{w}}$. Monodisperse HA was used as a standard; these details have been reported in our previous study [22].

\section{Chondroitin Digestion by Chondroitinase ABC and Mass Spectra Analysis}

To digest chondroitin, $1 \mathrm{ml}$ product solution $(1 \mathrm{mg} / \mathrm{ml})$ was treated with $1 \mathrm{IU}$ of chondroitinase $\mathrm{ABC}$ (Bicheng Biotech Co., Ltd., China) at $25^{\circ} \mathrm{C}$. Triple quadrupole liquid chromatographymass spectrometry (Shimadzu, Japan) was applied to analyze the disaccharide unit with electrospray ionization mass spectrometry (ESI-MS) in negative scan mode. MS/MS negative scan mode was further used to analyze monosaccharide fragments from peak $\mathrm{m} /$ $\mathrm{z}=378$.

\section{Shaking Flask Culture of Recombinant C. glutamicum}

Seed culture was conducted in LBG20 (yeast extract $5 \mathrm{~g} / \mathrm{l}$, peptone $10 \mathrm{~g} / \mathrm{l}, \mathrm{NaCl} 10 \mathrm{~g} / \mathrm{l}$, and glucose $20 \mathrm{~g} / \mathrm{l}$ ) liquid medium with $5 \mu \mathrm{g} / \mathrm{ml}$ chloramphenicol at $30^{\circ} \mathrm{C}, 200 \mathrm{rpm}$ overnight. When the $\mathrm{OD}_{600}$ reached $2.5,5 \% \mathrm{v} / \mathrm{v}$ inoculums were added to a $300-\mathrm{ml}$ flask containing $50 \mathrm{ml}$ modified culture medium [22] (glucose $40 \mathrm{~g} / \mathrm{l}$, corn syrup powder $20 \mathrm{~g} / \mathrm{l},\left(\mathrm{NH}_{4}\right)_{2} \mathrm{SO}_{4} 30 \mathrm{~g} / \mathrm{l}, \mathrm{KH}_{2} \mathrm{PO}_{4} 1 \mathrm{~g} / \mathrm{l}$, $\mathrm{K}_{2} \mathrm{HPO}_{4} \quad 0.5 \mathrm{~g} / \mathrm{l}, \quad \mathrm{MgSO}_{4} \quad 5 \mathrm{~g} / \mathrm{l}, \quad \mathrm{FeSO}_{4} \bullet 7 \mathrm{H}_{2} \mathrm{O} \quad 10 \mathrm{mg} / \mathrm{l}$, and $\mathrm{MnSO}_{4} \bullet 7 \mathrm{H}_{2} \mathrm{O} 10 \mathrm{mg} / \mathrm{l}$ ) with $5 \mu \mathrm{g} / \mathrm{ml}$ chloramphenicol. Shaking flask culture was performed at $28^{\circ} \mathrm{C}, 200 \mathrm{rpm}$, and $1 \mathrm{mM}$ isopropyl- $\beta$-D-thiogalactoside (IPTG) was added at $3 \mathrm{~h}$ to induce gene expression. C. glutamicum containing empty plasmid pXMJ19 was used as a control.

\section{GFPuv Reporter Assessment}

Six engineered strains containing different promoters (i.e., Pddh, PdapA, Psod, Pfba, Ptuf, and Ptac) were constructed with pECXK99E plasmid for promoter strength analysis. UV light-excited green fluorescent protein (GFPuv) was used as a reporter gene [27]. Briefly, seed cultures of six recombinant strains were conducted in LBG20 liquid medium with $50 \mu \mathrm{g} / \mathrm{ml}$ kanamycin at $30^{\circ} \mathrm{C}, 200 \mathrm{rpm}$ overnight. Then, $5 \% \mathrm{v} / \mathrm{v}$ inoculums were added to the $300-\mathrm{ml}$ flask containing $50 \mathrm{ml}$ modified culture medium [19] (for Ptac-GFPuv, $1 \mathrm{mM}$ IPTG was added at $3 \mathrm{~h}$ ). After $24 \mathrm{~h}$, cells were harvested at $4^{\circ} \mathrm{C}, 10,000 \times \mathrm{g}$. The pellets were resuspended in deionized water to $\mathrm{OD}_{600}=1.0$ and GFPuv expression intensities were monitored on a TECAN Infinite M200 PRO microplate reader (Männedorf, Switzerland). Wild-type C. glutamicum ATCC13032 was used as a control. Absorbance was measured at $385 \mathrm{~nm}$ and $509 \mathrm{~nm}$ extinction and emission wavelengths, respectively. The native promoter sequences were referenced from a previous promoter study [28].

\section{SDS-PAGE of Target Protein}

The $24 \mathrm{~h}$ fermentation sample taken from the shaking flask culture was centrifuged at $4^{\circ} \mathrm{C}, 10,000 \times g$ to collect cell pellets. The cell disrupter described earlier was used to release the whole cell proteins of recombinant C. glutamicum. Then, the sample was loaded onto Coomassie-stained SDS-PAGE to verify target protein expression.

RNA Isolation, Reverse Transcriptase PCR and Real-Time Quantitative PCR (qRT-PCR)

Cell pellets were collected from shaking flask culture at $24 \mathrm{~h}$ when the recombinant strains were in mid-exponential phase. An E.Z.N.A. Bacterial RNA Kit (Omega, USA) was applied to extract total RNA and reverse transcription using a PrimeScript 1st Strand cDNA Synthesis Kit (TaKaRa, China) to generate a cDNA library. Target gene transcript levels were quantified by qRT-PCR using the ABI 7300 real-time PCR system (Applied Biosystems, USA) and AceQ qPCR SYBR Green Master Mix (Vazyme, China) using primers qU-F and qU-R. Gene dnaE was used to normalize the target gene transcription level using qE-F and qE-R. Each experiment was performed in triplicate. Samples without reverse transcriptase treatment were used as negative controls to eliminate interference caused by DNA contamination.

\section{Fed-Batch Culture in a 5-L Fermentor}

A fed-batch culture of engineered C. glutamicum was performed in a 5-L fermentor (Sartorius stedim, BIOSTAT B plus) with a 2-L working volume. Aeration and agitation were set at $1 \mathrm{vvm}$ and $600 \mathrm{rpm}$, respectively. Briefly, $6 \mathrm{M} \mathrm{NaOH}$ and $6 \mathrm{HCl}$ were used to maintain fermentation $\mathrm{pH}$ at 7.2. A $5 \% \mathrm{v} / \mathrm{v}$ inoculum was prepared, and the fermentation temperature was controlled at $28^{\circ} \mathrm{C}$. At $3 \mathrm{~h}, 1 \mathrm{mM}$ IPTG was added to induce gene expression.

\section{Results}

\section{Construction of Chondroitin Biosynthesis Pathway in C. glutamicum}

Based on the biosynthesis mechanism of chondroitin in E. coli K4, we designed a biosynthesis pathway in C. glutamicum as shown in Fig. 1. Heterologous UDP-Nacetylglucosamine-4-epimerase (KfoA) and chondroitin polymerase $(\mathrm{KfoC})$ from E. coli $\mathrm{K} 4$ were introduced into the engineered C. glutamicum to generate the extra precursor UDP-GalNAc. Then, the chondroitin polymer was fabricated with another native precursor UDP-GlcUA. 


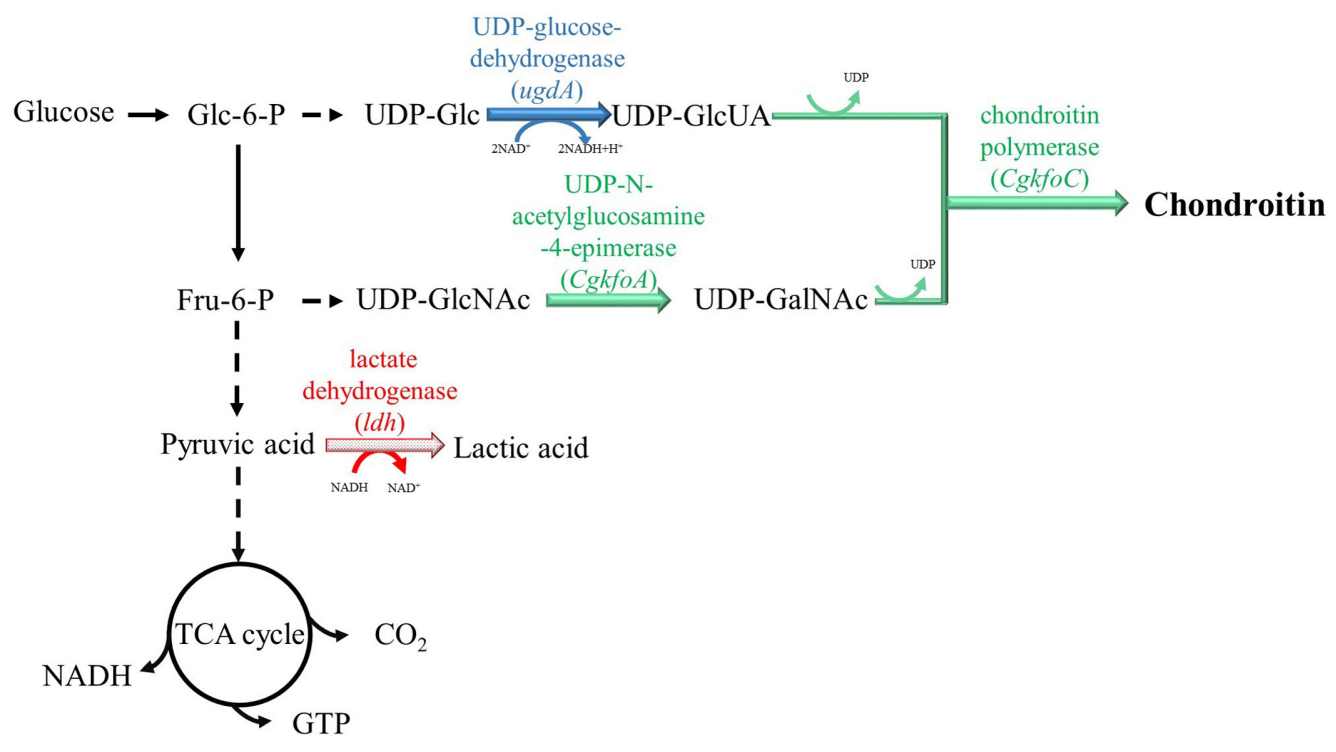

Fig. 1. Chondroitin biosynthesis pathway starting from glucose in recombinant C. glutamicum.

Heterologous genes $(C g k f o C$ and $C g k f o A)$ are marked in green bold arrow. Native gene $(u g d A)$ is marked in blue bold arrow. Byproduct synthesis gene $(l d h)$ is marked in red dot arrow. Dashed line represents multiple enzyme reactions.

Codon usage preference analyses showed that the original E. coli $\mathrm{K} 4 \mathrm{kfoC}$ gene (687 codons) contained 37 rare codons (usage frequency, $10-20 \%$ ) and 38 highly rare codons (usage frequency $<10 \%$ ) of C. glutamicum. The E. coli K4 $k f o A$ (340 codons) contained 21 rare codons and 17 highly rare codons of C. glutamicum (Fig. S2). To eliminate any possible negative effects from these rare codons, two new genes, $C g k f \circ C$ and $C g k f o A$, were designed and synthesized with a codon preference for C. glutamicum (Fig. S3).

The promoter is another key factor determining the overexpression efficiency of heterologous genes. Using the same plasmid vector, five native promoters (Pddh, PdapA, $\mathrm{P} f b a, \mathrm{Psod}$, and Ptuf) and one inducible strong promoter Ptac were compared with green fluorescent protein as a reporter. An optimal C. glutamicum RBS, AAAGGAGGA [29], was also applied for GFPuv overexpression. As shown
A

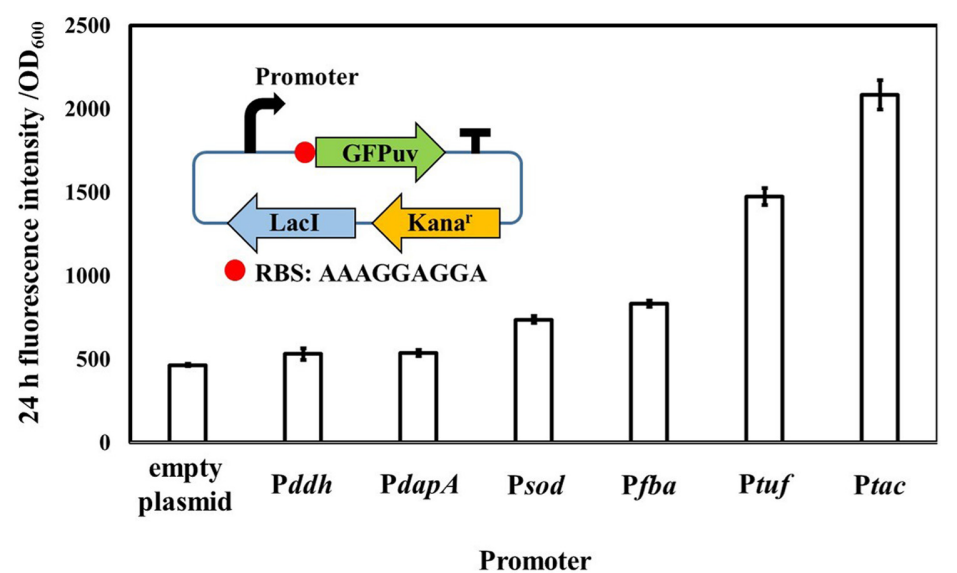

B

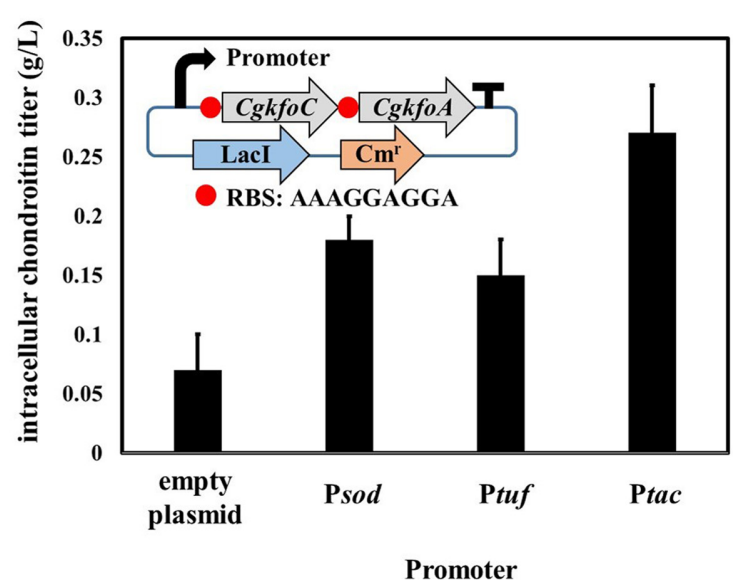

Fig. 2. (A) GFPuv reporter analysis of 5 native promoters and one inducible promoter Ptac in C. glutamicum. Pddh, PdapA, Psod, $\mathrm{Pfba}$, and Ptuf, promoters of diaminopimelate dehydrogenase, 4-hydroxy-tetrahydrodipicolinate synthase, superoxide dismutase, fructose 1,6 bisphosphate aldolase and translational elongation factor EF-Tu. Ptac, chimeric promoter of E. coli. (B) Chondroitin titers of recombinant C. glutamicum harboring $C g k f o C$ and $C g k f o A$ driven by Ptac, Ptuf, and Psod. 
in Fig. 2A, Ptac and Ptuf were strong promoters, and Psod and $\mathrm{P} f b a$ were moderately strong promoters in C. glutamicum. Furthermore, plasmid pXMJ19 harboring Ptac-CgKfoCA, Ptuf-CgKfoCA and Psod-CgKfoCA were transformed into C. glutamicum. Their chondroitin titers were measured with empty plasmid strain (Fig. 2B). Thus, inducible promoter Ptac was an ideal promoter for expressing the $k f o$ genes in recombinant C. glutamicum.

Lactic acid synthesis, catalyzed by lactate dehydrogenase (LDH), dissipates the energy produced from GAG production. In our previous study, deletion of gene $l d h$ effectively increased ATP and carbon flux redistribution to GAG production [23]. Herein, gene ldh knockout was applied (yielding $l d h$-knockout strain C. glutamicum- $\Delta l d h$ ) to evaluate and compare its impact on chondroitin synthesis with wild-type host C. glutamicum ATCC 13032. The recombinant plasmid pXMJ19-Ptac-CgKfoCA was transformed into both C. glutamicum ATCC 13032 and C.glutamicum$\Delta l d h$, yielding the engineered strains $C$. glutamicum$\mathrm{CgKfoCA}(\mathrm{CgCA})$ and C. glutamicum- $\Delta l d h-C g \mathrm{KfoCA}(\Delta \mathrm{L}-$
A

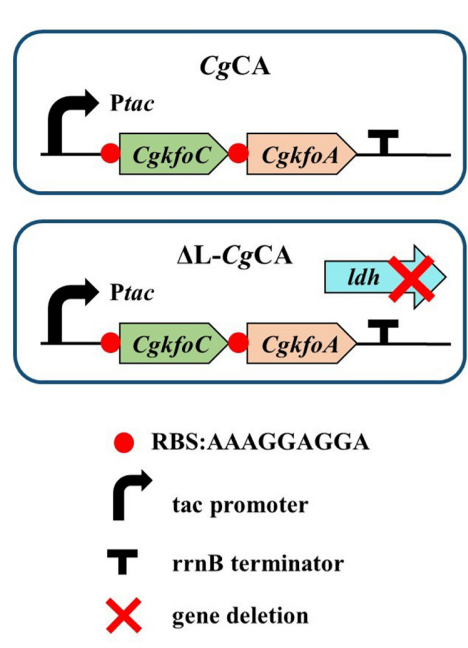

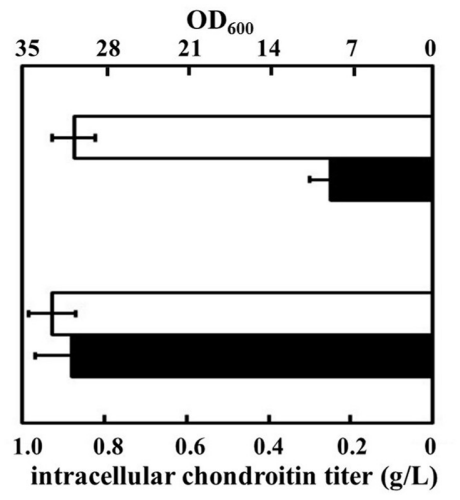

intracellular chondroitin titer $\square$ OD $_{600}$

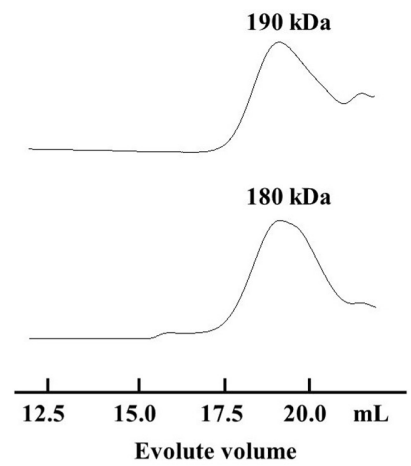

B

chondroitinase
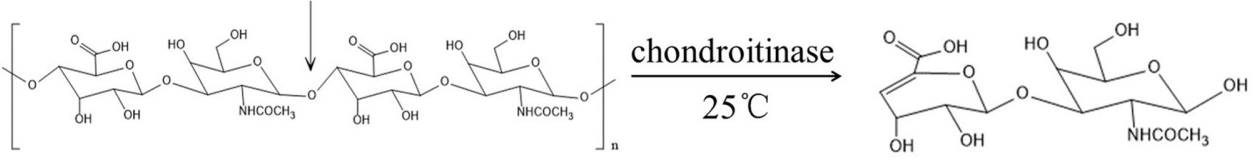

\section{C}
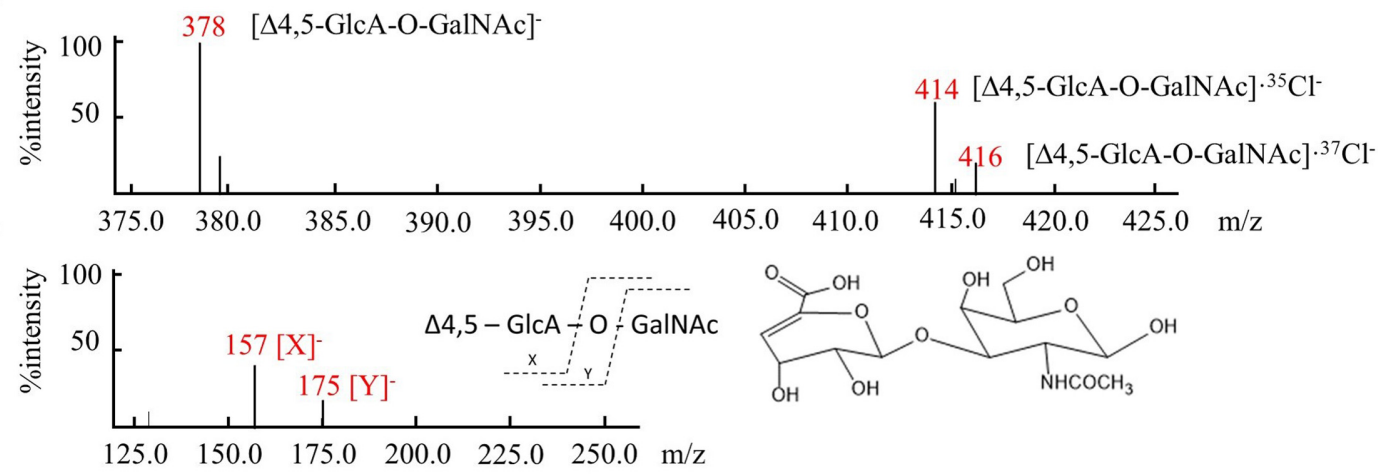

Fig. 3. Chondroitin biosynthesis in recombinant C. glutamicum $\mathrm{CgCA}$ and $\Delta \mathrm{L}-\mathrm{CgCA}$.

(A) Intracellular chondroitin titer, $\mathrm{OD}_{600}$ and $\mathrm{M}_{\mathrm{w}}$ of control strain (with empty plasmid), $\mathrm{Cg} \mathrm{CA}$ and $\Delta \mathrm{L}-\mathrm{Cg} \mathrm{CA}$ in shaking flask culture. (B) Chondroitinase ABC hydrolysis reaction. (C) ESI-MS result of the digested chondroitin product in negative scan mode. (D) MS/MS result of peak $\mathrm{m} / \mathrm{z}=378$ in negative scan mode. The target peaks were labeled with its $\mathrm{m} / \mathrm{z}$ and molecular formula. Each experiment was performed in triplicate. 
$\mathrm{Cg} \mathrm{CA})$. Successful expressions of $\mathrm{KfoC}$ and $\mathrm{KfoA}$ in C. glutamicum were verified by SDS-PAGE (Fig. S4).

Shaking flask culture was performed in modified medium to investigate the chondroitin production capability of $\mathrm{CgCA}$ and $\Delta \mathrm{L}-\mathrm{CgCA}$. As shown in Fig. 3A, the engineered strain $\mathrm{CgCA}$ accumulated $0.25 \mathrm{~g} / 1$ intracellular chondroitin, whereas the $l d h$-knockout significantly increased the chondroitin titer to $0.88 \mathrm{~g} / \mathrm{l}$ with nearly the same $\mathrm{M}_{\mathrm{w}}$ (180$190 \mathrm{kDa})$. The molecular structure of chondroitin was verified by chondroitinase $\mathrm{ABC}$ digestion (Fig. 3B) and subsequent ESI-MS (Fig. 3C) and MS/MS (Fig. 3D) analyses of the digested products. As shown in Fig. 3C, disaccharide units $[\Delta 4,5-\mathrm{GlcA}-\mathrm{O}-\mathrm{GalNAc}]^{-}$, [ $\left.\Delta 4,5-\mathrm{GlcA}-\mathrm{O}-\mathrm{GalNAc}\right] \cdot{ }^{35} \mathrm{Cl}^{-}$ and $[\Delta 4,5-\mathrm{GlcA}-\mathrm{O}-\mathrm{GalNAc}]{ }^{37} \mathrm{Cl}^{-}$were identified. As shown in Fig. 3D, two monosaccharide fragments were observed by MS/MS. No extracellular chondroitin was obtained, however, indicating that the extracellular transport of chondroitin was restricted in C. glutamicum.

\section{Enhanced Biosynthesis of Chondroitin via ugdA Overexpression}

According to studies on the microbial production of GAG (e.g., hyaluronic acid and chondroitin), UDP-glucose dehydrogenase was identified as a rate-limiting factor in the synthesis pathway $[12,16,23,30]$. Gene $u g d A$ expression was up-regulated to enhance the biosynthesis of UDPGlcUA, another chondroitin precursor (Fig. 1). The native ugdA of C. glutamicum was cloned and integrated into pXMJ19-Ptac-CgKfoCA, yielding pXMJ19-Ptac-CgKfoCAU. Recombinant plasmid pXMJ19-Ptac-CgKfoCAU was transformed into wild-type and $l d h$-knockout strains to obtain two new strains: C. glutamicum-CgKfoCAF (CgCAU) and C. glutamicum- $\Delta l d h-C g K f o C A U ~(\Delta L-C g C A U)$ (Fig. $4 \mathrm{~A})$. The qRT-PCR results confirmed that the transcript levels of $u g d A$ in $C g C A U$ and $\triangle \mathrm{L}-C g C A U$ were significantly enhanced (over 20-fold after accounting for DNA interference) after ugdA overexpression with Ptac promoter (Fig. 4B). Chondroitin
A

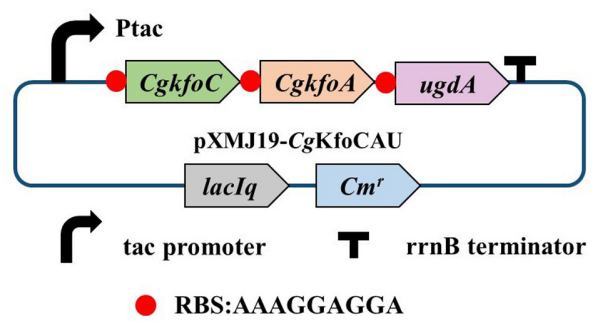

C

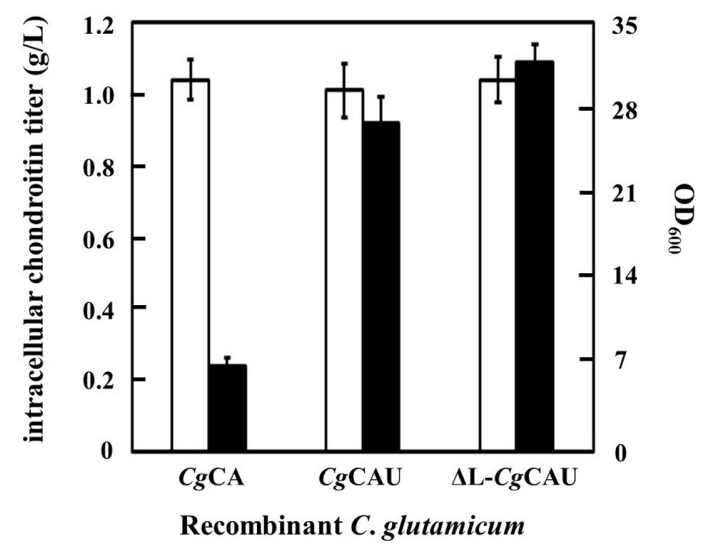

intracellular chondroitin titer

$\square$ OD $_{600}$
B

\begin{tabular}{cccc}
\hline $\begin{array}{c}\text { Recombinant } \\
\text { strain }\end{array}$ & $C g \mathrm{CA}$ & $\operatorname{CgCAU}$ & $\Delta \mathrm{L}-\mathrm{CgCAU}$ \\
\hline $\begin{array}{c}\text { Relative } \\
\text { expression level } \\
\text { (with reverse } \\
\text { transcriptase) }\end{array}$ & $1.0 \pm 0.3$ & $24.7 \pm 3.5$ & $29.3 \pm 4.1$ \\
\hline $\begin{array}{c}\text { Relative } \\
\text { expression level } \\
\text { (without reverse } \\
\text { transcriptase) }\end{array}$ & $0.02 \pm 0.01$ & $0.18 \pm 0.05$ & $0.20 \pm 0.08$ \\
\hline
\end{tabular}

D

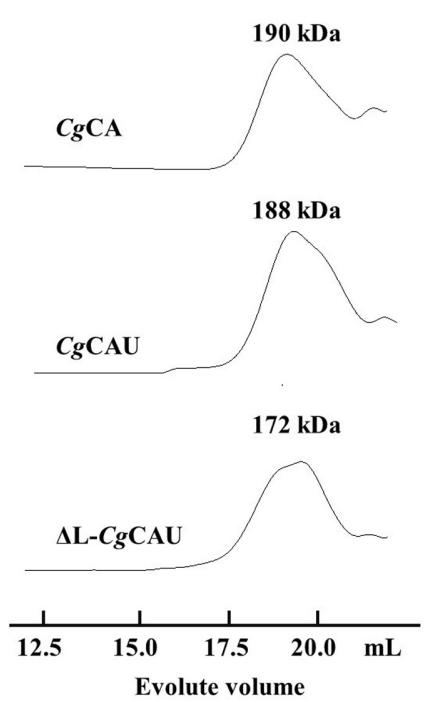

Fig. 4. Chondroitin biosynthesis in $u g d A$ overexpressed recombinant $C$. glutamicum $C g \mathrm{CAU}$ and $\Delta \mathrm{L}-\mathrm{CgCAU}$.

(A) Plasmid structure of pXMJ19-CgKfoCAU (B) qRT-PCR results of gene ugdA transcription in different engineered strains. (C) Intercellular chondroitin titer and $\mathrm{OD}_{600}$ of $C g \mathrm{CA}, \mathrm{CgCAU}$ and $\Delta \mathrm{L}-\mathrm{CgCAU}$. (D) Chondroitin's $\mathrm{M}_{\mathrm{w}}$. Experiments were performed in triplicate. 
titers from these two strains were compared in shaking flask culture using $\mathrm{Cg} C \mathrm{CA}$ as a control. As illustrated in Fig. $4 \mathrm{C}$, the overexpression of $u g d A$ in a wild-type host increased the chondroitin titer from $0.24 \mathrm{~g} / 1$ to $0.92 \mathrm{~g} / \mathrm{l}$, whereas in the $l d h$ deletion strain $\Delta \mathrm{L}-\mathrm{CgCAU}$, the chondroitin titer was $1.09 \mathrm{~g} / 1$ (Fig. 4C). Similar $\mathrm{M}_{\mathrm{w}}$ distributions (172, 188, and $190 \mathrm{kDa}$, respectively) were also observed for the engineered strains (Fig. 4D). Collectively, strain $\triangle \mathrm{L}-\mathrm{Cg} \mathrm{CAU}$ showed the highest chondroitin titer and thus, was chosen for further investigation in the fed-batch culture.

\section{Fed-Batch Culture of Recombinant $\Delta \mathrm{L}-\mathrm{CgCAU}$ in a 5 - $\mathrm{L}$ Fermentor}

Because chondroitin consists of multiple disaccharide units, glucose was fed to ensure a sufficient carbon source for chondroitin production. As shown in Fig. 5A, we continuously pumped glucose into the fermentor to maintain glucose concentration at $8-15 \mathrm{~g} / \mathrm{l}$ from $17 \mathrm{~h}$ until the end of the cultivation (Fig. 5A).

During the fed-batch culture, a maximum $\mathrm{OD}_{600}$ of recombinant strain $\triangle \mathrm{L}-\mathrm{CgCAU}$ reached 101, indicating the start of the stationary phase at $20 \mathrm{~h}$ (Fig. 5B). The maximum chondroitin titer reached $1.91 \mathrm{~g} / \mathrm{l}$ at $28 \mathrm{~h}$. Productivity was calculated as $0.068 \mathrm{~g} / \mathrm{l} / \mathrm{h}$ at this time. GPC analysis showed that the $\mathrm{M}_{\mathrm{w}}$ of the chondroitin product remained stable at approximately $186 \mathrm{kDa}$ throughout the fermentation (Fig. 5C).

\section{Discussion}

With the development of biotechnology and increasing health concerns regarding animal-derived products, the microbial production of chondroitin shows great potential for producing CS. A safer but efficient host is an important property for the microbial production of chondroitin. As reported in the literature, engineered B. subtilis 168 has been the most successful host in producing chondroitin [16, 17]. In this work, however, C. glutamicum was utilized for the first time, and its capability to produce chondroitin was explored via several engineered strategies.

The chondroitin synthesis pathway of E. coli $\mathrm{K} 4$ was mimicked. Two key enzymes, KfoC and KfoA for synthesizing chondroitin, were overexpressed in C. glutamicum. Two novel genes, $\mathrm{CgkfoC}$ and $\mathrm{CgkfoA}$, were synthesized after codon preference optimization. The optimal strong promoter, Ptac, was selected for the overexpression of $\mathrm{CgkfoC}$ and $C g k f o A$ and compared with 5 other widely used promoters, such as Ptuf and Psod of C. glutamicum; Ptuf is generally

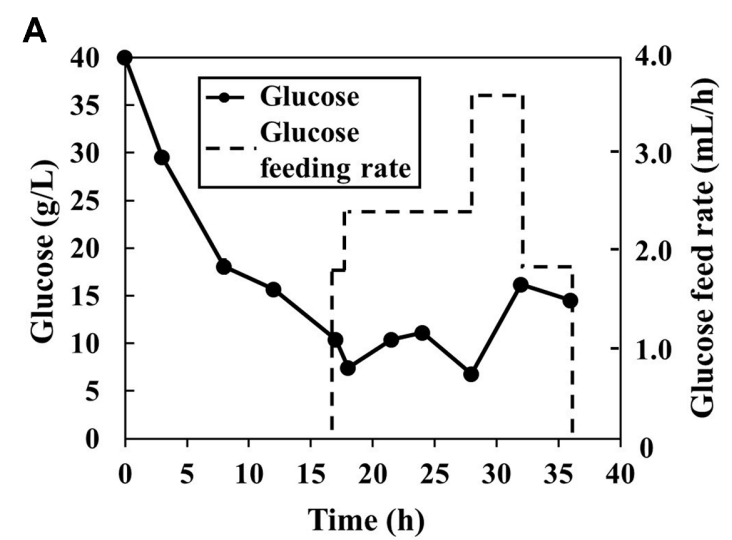

B
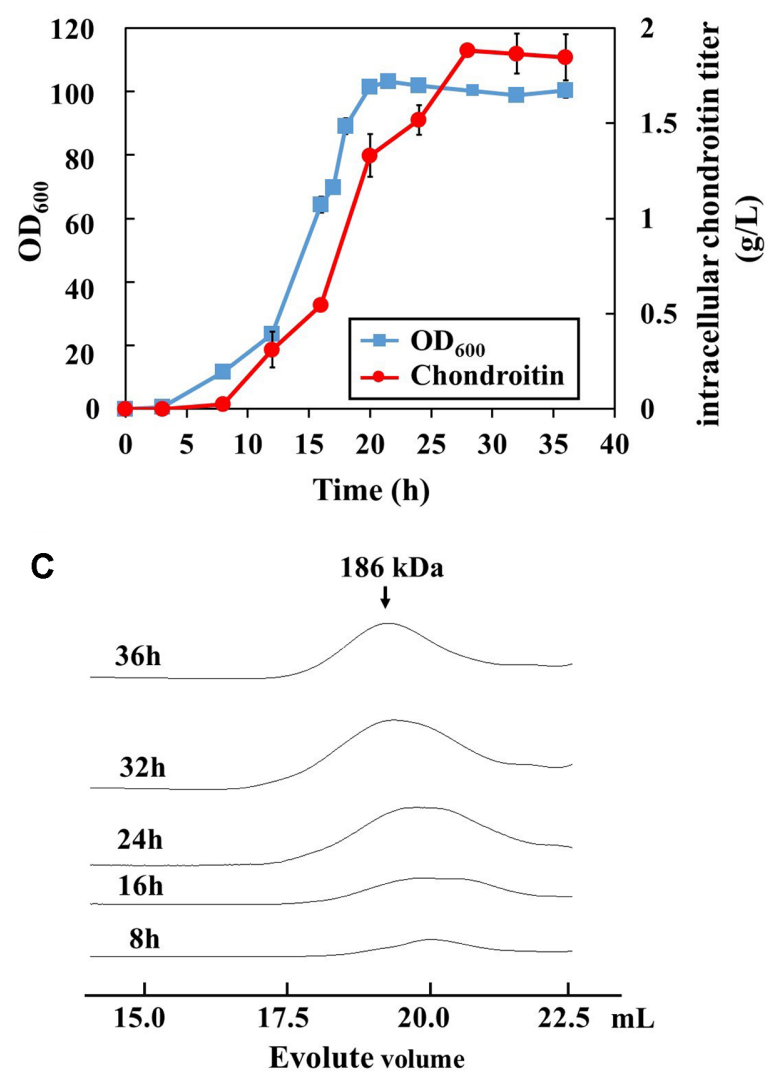

Fig. 5. Fed-batch culture of $\Delta \mathrm{L}-\mathrm{Cg} C \mathrm{CAU}$ in a 5 -L fermentor. (A) Glucose concentration (solid line) and feeding rate (dashed line). (B) $\mathrm{OD}_{600}$ and chondroitin titer. (C) $\mathrm{M}_{\mathrm{w}}$ of chondroitin. Experiments were performed in duplicate.

acknowledged as a strong promoter $[28,31]$. The GFPuv reporter assessment results showed that Ptac was more efficient than the native strong promoter Ptuf [28]. Furthermore, $\mathrm{CgkfoC}$ and $\mathrm{CgkfoA}$ co-expression driven by Ptac generated $0.25 \mathrm{~g} / 1$ chondroitin in the engineered C. glutamicum. 
Carbon flux redirection is an effective method for intensifying GAG production. For instance, HA titer was promoted via ldh deletion in L. lactis and C. glutamicum [23, 32]. Its effect also embodied in redox balance [32] and enhancing ATP supplementation [23]. Herein, an LDH knockout C. glutamicum host was applied to enhance chondroitin production. The chondroitin titer increased to $0.88 \mathrm{~g} / \mathrm{l}$, illustrating that deleting $l d h$ was effective to enhance chondroitin biosynthesis.

An over-expressing of the enzymes responsible for the biosynthesis of UDP-sugar precursors from carbon source is another major approach for enhancing GAG production. Previous studies have demonstrated that UDP-glucose dehydrogenase (KfoF in E.coli K4, KfiD in E.coli K5, HasB in S. equi, TuaD in B. subtilis and UgdA in C. glutamicum) in the GAG biosynthesis pathway is a rate-limiting enzyme and is strictly regulated in UDP-GlcUA biosynthesis [22, 33]. For example, in a study of hyaluronic acid production by recombinant $C$. glutamicum, the optimal operon structure was hasA-hasB [22, 23]. Similar strategies have also been applied in the heterogeneous production of heparson, another GAG with a similar structure as $\mathrm{HA}$ and chondroitin [16]. The overexpression of $t u a D$ (an encoding isozyme of $\mathrm{KfoF}$ ) in recombinant $B$. subtilis also resulted in a promotion of chondroitin titer from $1.63 \mathrm{~g} / 1$ to $2.36 \mathrm{~g} / 1$ [16]. In this study, precursor UDP-GlcUA was enhanced via the overexpression of $u g d A$ with strong promoter Ptac. The chondroitin titer was enhanced more than 3-fold, indicating that UDP-glucose dehydrogenase is a rate-limit enzyme as well in chondroitin biosynthesis of engineered C. glutamicum.

Molecular weight is another important property of microbial-produced chondroitin. In the study of recombinant B. subtilis, the $\mathrm{M}_{\mathrm{w}}$ of chondroitin increased from $84 \mathrm{kDa}$ to $114 \mathrm{kDa}$ after UDP-glucose dehydrogenase upregulation, but decreased to $66 \mathrm{kDa}$ after the culture mode changed from shaking flask to 3-L fermentor. Jin et al. attributed the decrease to shearing forces caused by mechanical agitation [16]. In this work, however, the $\mathrm{M}_{\mathrm{w}}$ from different strains or different culture modes were all approximately $180 \mathrm{kDa}$. Because nearly all chondroitin products were retained in the cell pellet, the cell membrane and walls may shield chondroitin from external factors. Due to the negative relation between GAG titer and its $\mathrm{M}_{\mathrm{w}}$ in HA production $[34,35]$, this high $\mathrm{M}_{\mathrm{w}}$ may contribute to the relatively low chondroitin titer in the engineered C. glutamicum.

Further research on chondroitin titer enhancement is still required. A more comprehensive operon structure that takes both UDP-GalNAC and UDP-GlcUA into consideration and an efficient transport system that can obtain extracellular chondroitin or other metabolic or transcriptional regulations are feasible strategies for engineered C. glutamicum.

\section{Acknowledgment}

This work was supported by the National Natural Science Foundation (No. 21776157) and the National Key Basic Research Project 973 (2013CB733600) of China.

\section{Conflict of Interest}

The authors have no financial conflicts of interest to declare.

\section{References}

1. Vázquez JA, Rodríguezamado I, Montemayor MI, Fraguas J, González MDP, Murado MA. 2013. Chondroitin sulfate, hyaluronic acid and chitin/chitosan production using marine waste sources: characteristics, applications and ecofriendly processes: a review. Mar. Drugs. 11: 747-774.

2. Schiraldi C, Cimini D, De Rosa M. 2010. Production of chondroitin sulfate and chondroitin. Appl. Microbiol. Biotechnol. 87: 1209-1220.

3. Roman E, Roberts I, Lidholt K, Kusche-Gullberg M. 2003. Overexpression of udp-glucose dehydrogenase in Escherichia coli results in decreased biosynthesis of $\mathrm{k} 5$ polysaccharide. Biochem. J. 374: 767-772.

4. Kwok JC, Warren P, Fawcett JW. 2012. Chondroitin sulfate: a key molecule in the brain matrix. Int. J. Biochem. Cell Biol. 44: 582-586.

5. Lauder R. 2009. Chondroitin sulphate: a complex molecule with potential impacts on a wide range of biological systems. Complement. Ther. Med. 17: 56-62.

6. Mikami T, Kitagawa H. 2013. Biosynthesis and function of chondroitin sulfate. BBA-Gen. Subjuects 1830: 4719-4733.

7. He W, Zhu Y, Shirke A, Sun X, Liu J, Gross RA, Koffas MAG, Linhardt RJ, Li M. 2017. Expression of chondroitin-4O-sulfotransferase in Escherichia coli and Pichia pastoris. Appl. Microbiol. Biotechnol. 101: 6919-6928.

8. Badri A, Williams A, Linhardt R J, Koffas M A G. 2017. The road to animal-free glycosaminoglycan production: current efforts and bottlenecks. Curr. Opin. Biotechnol. 53: 85-92.

9. Williams A, Linhardt R J, Koffas M A G. 2018. Metabolic engineering of capsular polysaccharides. Emerg Top Life Sci. 2: 337-348.

10. Rodriguez ML, Jann B, Jann K. 2010. Structure and serological characteristics of the capsular k4 antigen of escherichia coli, O5:K4:H4, a fructose-containing polysaccharide with a chondroitin backbone. Eur. J. Biochem. 177: 117-124. 
11. Bedini E, De Castro C, De Rosa M, Di Nola A, Iadonisi A, Restaino OF, et al. 2011. A microbiological-chemical strategy to produce chondroitin sulphate A, C. Angew. Chem. Int. Ed. Engl. 50: 6160-6163.

12. Cimini D, Fantaccione S, Volpe F, De RM, Restaino OF, Aquino G. 2014. IS2-mediated overexpression of Kfoc in E. coli K4 increases chondroitin-like capsular polysaccharide production. Appl. Microbiol. Biotechnol. 98: 3955-3964.

13. Zhang Q, Yao R, Chen X, Liu L, Xu S, Chen J. 2018. Enhancing fructosylated chondroitin production in Escherichia coli $\mathrm{K} 4$ by balancing the UDP-precursors. Metab. Eng. 47: 314-322.

14. Wiles TJ, Kulesus RR, Mulvey MA. 2008. Origins and virulence mechanisms of uropathogenic Escherichia coli. Exp. Mol. Pathol. 85: 11-19.

15. He W, Fu L, Li G, Andrew JJ, Linhardt RJ, Koffas M. 2015. Production of chondroitin in metabolically engineered E. coli. Metab. Eng. 27: 92-100.

16. Jin P, Zhang L, Yuan P, Kang Z, Du G, Chen J. 2016. Efficient biosynthesis of polysaccharides chondroitin and heparosan by metabolically engineered Bacillus subtilis. Carbohydr. Polym. 140: 424-432.

17. Zhou Z. Li Q, Huang H, Wang H, Wang Y, Du G, Chen J, Kang Z. 2018. A microbial-enzymatic strategy for producing chondroitin sulfate glycosaminoglycans. Biotechnol. Bioeng. 115: $1561-1570$.

18. Guo Y, Han M, Yan W, Xu J, Zhang W. 2014. Generation of branched-chain amino acids resistant Corynebacterium glutamicum, acetohydroxy acid synthase by site-directed mutagenesis. Biotechnol. Bioprocess Eng. 19: 456-467.

19. Neshat A, Mentz A, Rückert C, Kalinowski J. 2014. Transcriptome sequencing revealed the transcriptional organization at ribosome-mediated attenuation sites in Corynebacterium glutamicum and identified a novel attenuator involved in aromatic amino acid biosynthesis. J. Biotechnol. 190: 55-63.

20. Xu JZ, Wu ZH, Gao SJ, Zhang W. 2018. Rational modification of tricarboxylic acid cycle for improving 1 lysine production in Corynebacterium glutamicum. Microb. Cell Fact. 17: 105.

21. Zhang $Y$, Shang X, Lai S, Zhang $Y, H u$ Q, Chai X, et al. 2018. Reprogramming one-carbon metabolic pathways to decouple 1-serine catabolism from cell growth in Corynebacterium glutamicum. Acs Synth. Biol. 101: 1-12.

22. Cheng F, Gong Q, Yu H, Stephanopoulos G. 2016. High-titer biosynthesis of hyaluronic acid by recombinant Corynebacterium glutamicum. Biotechnol. J. 11: 574-584.
23. Cheng F, Luozhong S, Guo Z, Yu H, Stephanopoulos G. 2017. Enhanced biosynthesis of hyaluronic acid using engineered Corynebacterium glutamicum via metabolic pathway regulation. Biotechnol. J. 12: 1700268.

24. Sambrook J, Fritsch EF, Maniatis T. 1988. Molecular cloning: A laboratory manual, 2nd edition. Cold Spring HarborLaboratory Press, Cold Spring Harbor, NY.

25. Schafer A, Tauch A, Jager W, Kalinowski J, Thierbach G, Puhler A. 1994 Small mobilizable multi-purpose cloning vectors derived from the Escherichia coli plasmids pK18 and pK19: selection of defined deletions in the chromosome of Corynebacterium glutamicum. Gene 145: 69-73.

26. Bitter T, Muir HM. 1962. A modified uronic acid carbazole reaction. Anal. Biochem. 4: 330-334.

27. Crameri A, Whitehorn EA, Tate E, Stemmer WP. 1996. Improved green fluorescent protein by molecular evolution using DNA shuffling. Nat. Biotechnol. 14: 315-319.

28. Shang X, Chai X, Lu X, Li Y, Zhang Y, Wang, G, et al. 2018. Native promoters of Corynebacterium glutamicum and its application in L-lysine production. Biotechnol. Lett. 40: 383-391.

29. Liu Q, Ouyang S, Kim J, Chen G. 2007. The impact of PHB accumulation on L-glutamate production by recombinant Corynebacterium glutamicum. J. Biotechnol. 123: 273-279.

30. Cimini D, Carlino E, Giovane A, Argenzio O, Dello Iacono I, De Rosa M, et al. 2016. Engineering a branch of the UDPprecursor biosynthesis pathway enhances the production of capsular polysaccharide in Escherichia coli O5:K4:H4. Biotechnol. J. 33: 1307-1315.

31. Pátek M, Holátko J, Busche T, Kalinowski J, Nešvera J. 2013. Corynebacterium glutamicum promoters: a practical approach. Microb. Biotechnol. 6: 103-117.

32. Kaur M., Jayaraman G. 2016. Hyaluronan production and molecular weight is enhanced in pathway-engineered strains of lactate dehydrogenase-deficient Lactococcus lactis. Metab. Eng. Commun. 3: 15-23.

33. Widner B, Behr R, Von Dollen S, Tang M, Heu T, Sloma A, et al. 2005. Hyaluronic acid production in Bacillus subtilis. Appl. Environ. Microbiol. 71: 3747-3752.

34. Westbrook AW, Ren X, Oh J, Mooyoung M, Chou CP. 2018. Metabolic engineering to enhance heterologous production of hyaluronic acid in Bacillus subtilis. Metab. Eng. 47: 401-413.

35. Yang J, Cheng F, Yu H, Wang J, Guo Z, Stephanopoulos G. 2017. Key role of the carboxyl terminus of hyaluronan synthase in processive synthesis and size control of hyaluronic acid polymers. Biomacromolecules 18: 1064-1073. 